\title{
Reference Intervals for Absolute and Percentage Immature Platelet Fraction using the Sysmex XN-10 Automated Haematology Analyser in a UK Population
}

\section{Usman Ali, Gavin Knight, Roz Gibbs and Dimitris A. Tsisikas}

\begin{abstract}
Background: Immature platelet fraction (IPF) estimation is a non-invasive and sensitive test that is available on recently introduced Sysmex XN-series of automated haematology analysers. It is a direct cellular indicator of thrombopoiesis. The aim of this study was to establish reference intervals for IPF, for both absolute (A-IPF) and percentage (\%-IPF) measurements. Materials and Methods: A total of 2366 samples that met the inclusion criteria were assayed for full blood count on the Sysmex $\mathrm{XN}-10$ and a non-parametric percentile method was used for calculating the reference intervals.
\end{abstract}

Results: After the outliers were excluded, the reference interval for \%-IPF and A-IPF on Sysmex $\mathrm{XN}-10$ were $1.6-10.1 \%$ and $4.37-23.21 \times 10^{9} / \mathrm{l}$ in total individuals, respectively. There was a statistical significance noted between the sexes $(P=0.004)$ for $\%$-IPF, therefore a sex-specific reference interval was established, which was $1.8-10.0 \%$ for the males and $1.5-10.1 \%$ for females. No significant difference in sex status for A-IPF and age status for both \%-IPF and AIPF was observed. A very poor correlation was estimated between age versus $\%$-IPF, $\rho=0.0156$, and age versus A-IPF, $\rho=-0.0023$, indicating that there is no overall biological relationship between age and these parameters. As expected, a strong correlation between \%-IPF and A-IPF was noted which could be attributed to their inter-relatedness.

Conclusion: This large-scale study showed comparable reference intervals with the previous studies for \%-IPF and A-IPF in a UK population. It found the need to establish sex-specific reference intervals for \%-IPF, but not for A-IPF, whereas reference intervals were found to be stable across the age range. 
Keywords: reference values, blood platelet, haematology, thrombocytopenia, blood cell count, haematologic tests, haematologic diseases, clinical laboratory techniques, clinical laboratory services, United Kingdom

\section{Introduction}

Ingram and Coopersmith first showed that the number of immature platelets increase following acute blood loss [1]. Using a new methylene blue dye, these immature platelets demonstrated coarse, punctate, condensations [reticulum; thus called reticulated platelets (RP)] and are considered analogous to reticulocytes. Two decades later, it was reported that flow cytometry could detect platelet nucleic acid in RP using a dye that was named 'thiazole orange' [2]. Subsequent reports thus focused on flow cytometric analysis of these RNA-rich RPs using RNA binding dyes to provide information on thrombopoietic activity [3] in a variety of conditions such as: thrombocytopenia [4-10], thrombocytosis [11,12], thrombo-embolic disorders [13,14], hereditary platelet diseases [15,16], following stem cell transplantation [17-20], hyperthyroidism [21], kidney disease [22-24], preeclampsia [25], and thrombocytopenic and healthy neonates [26,27]. Accordingly, RP became reliably expressed as the immature platelet fraction (IPF) which replaced labour-intensive manual procedures and time consuming general flow cytometry. These studies concluded that IPF is a useful, non-invasive, marker for megakaryopoietic activity [28].

In current clinical practice, \%-IPF is measured by different haematology analysers ranging from Sysmex XE-series to XN-series [29]. The Sysmex XE-2100 (Sysmex, Kobe, Japan) was introduced in 1999 [30]. Subsequently, this analyser underwent a number of upgrades, including an automated

method for measuring \%-IPF, using flow cytometry [31]. In 2007, the Sysmex XE-5000 was 
launched with additional parameters [32]. A more advanced XN Modular analyser was introduced in 2011 which was similar to the previous XE-series instruments measuring red cell parameters and impedance platelets in the same way. Four new channels were introduced including a fluorescent platelet (PLT-F) channel which provided a better sensitivity and specificity for measuring \%-IPF compared to the previous versions of Sysmex haematology analysers [32]. IPF can be reported as both the percentage of immature platelets (\%-IPF), which the analyser generates, as well as absolute-IPF (A-IPF), which is a calculated measurement using both the platelet count and \%-IPF [33].

Earlier reference intervals that were established using the XE-series may be invalid for the XNseries of haematology analysers. More recent studies conducted in China [29] and Korea [34] have used the $\mathrm{XN}$-series, but have reported different reference intervals which may be associated with racial and ethnic variation. The aim of the present study, which is the largest study to date, was to establish new reference intervals for A-IPF and \%-IPF in a UK population, for the first time, using the Sysmex XN-10 automated haematology analyser.

\section{Materials and Methods}

\section{Sample Requirement and Inclusion Criteria}

Samples of venous whole-blood collected in ethylenediaminetetraacetic acid K2 (EDTA K2) Vacutainer tubes and received from primary care providers in the Department of Haematology at Homerton University Hospital were analysed on Sysmex XN-10. To minimise variations due to sample aging, all tests were completed within 12 hours of collection. A total of 2366 samples with routinely reported FBC parameters within the reference range were assayed by selecting PLT-F

mode on the Sysmex XN-10. All samples that met the following inclusion criteria were included 
in this study: (i) haemoglobin (Hb) 115-165 g/L [females] and 130-180 g/L [males]; (ii) red blood count (RBC) 3.80-5.80 x 10²/L [females] and 4.50-6.50 x 10²/L [males]; (iii) haematocrit (HCT) 0.37-0.47 L/L [females] and 0.40-0.54 L/L [males]; (iv) mean cell volume (MCV) 80-98 fL; (v) mean cell haemoglobin (MCH) 27-32 pg;(vi) mean cell haemoglobin concentration (MCHC) 310350 g/L; (vii) red blood cell distribution width (RDW) 10-14\%; (viii) platelet count (PLT) 150$400 \times 10^{9} / \mathrm{L}$; (ix) white blood count (WBC) 4.0-11.0 x 10\%/L; (x) neutrophil count (NEUT) 2.07.5 x 10\%/L; (xi) lymphocyte count (LYMPH) 1.0-4.0 x 10\%L; (xii) monocyte count (MONO) 0.2$1.0 \times 10^{9} / \mathrm{L}$; (xiii) eosinophil count (EO) 0.0-0.4 x 10\%/L; and (xiv) basophil count (BASO) 0.0$0.1 \times 10^{9} /$ L. Daily and weekly internal quality procedures were used for validating the quality of the results and imprecision was kept minimal with excellent intra-assay coefficients of variability (CV) of $<2 \%$ for 11 repeat analysis cycles (precision check). The study received a favourable opinion from the National Research Ethics Service (NRES).

\section{Measurement of immature platelets}

EDTA K2 anticoagulated, whole-blood, samples were measured on the Sysmex XN-10 analyser (Sysmex, Kobe, Japan) which is a widely used, routine, fully-automated haematology analyser. Compared to its previous versions, this analyser uses different principles, channels, and reagents with improved sensitivity and specificity for \%-IPF measurement [32]. In addition to impedance (PLT-I) and optic (PLT-O) methods for measuring platelet counts, the Sysmex XN-10 has a novel PLT-F-channel dedicated to measuring mature and immature platelets (Figure 1). This channel was introduced to improve the gating of platelets to achieve a more accurate platelet count (PLT-F), using the new fluorescent method, and \%-IPF. The analyser uses an algorithm to determine which platelet count to use - impedance (PLT-I), optical (PLT-O) or the fluorescent method (PLT-F). The measurement of IPF requires platelet cellular membranes to be perforated by reagents, viz. a fluorescent dye called oxadine, which stains the nucleic acids [32,35]. IPF enumeration is based on 
the principle of haemocytometry and is defined by an algorithm which gates for mature and immature platelets using side fluorescence (reflecting RNA content), side scatter (intracellular structure) and forward scatter (cell size) of light. An example of an IPF scattergram is shown in Figure 1. Immature platelets decrease in RNA content and size as they age, eventually becoming mature platelets [36]. It has been recently indicated that this RNA is used by platelets for protein synthesis [37].

\section{Immature platelets - absolute number (A-IPF) and percentage (\%-IPF)}

IPF is reported in two formats: as the percentage of platelets with above-threshold megakaryocytederived RNA (\%-IPF) - a value generated by XE- and XN-series automated haematology analysers - or the number of immature platelets per unit volume, known as the absolute-IPF (A-IPF). The A-

IPF can be calculated using the formula $\frac{\%-I P F \times \text { Platelet } \cdot \text { Count }}{100}$ [38]. Unlike \%-IPF, the A-IPF is the exact number of immature platelets in the circulation [33].

\section{Statistical analysis}

Data was recorded in Excel files. Outliers, defined as values $<$ Q1-1.5(IQR) or > Q3+1.5(IQR), [39] were checked and eliminated in addition to the data removed following application of the exclusion criteria. Statistical analysis was performed using Excel 2013 and RStudio (Version 0.99.903) to analyse and graphically represent the data. Histograms were used to determine the distribution of the data and a normality test was performed using the Kolmogorov-Smirnov test [40]. Reference intervals were calculated according to the Clinical and Laboratory Standard Institute (CLSI) guideline EP28-A3c [41]; the non-parametric percentile method (2.5th and 97.5th percentiles) was used to calculate the reference intervals for \%-IPF and A-IPF without making assumptions about the underlying distribution. This method is superior as no model-based data fitting is required [42]. Mann-Whitney Test was used to compare male and female counts only. 
Kruskal-Wallis one-way analysis of variance was used to compare the test parameters, \%-IPF and A-IPF, with seven age subgroups mainly by decades including: 16-19 years; 20-29 years; 30-39 years; 40-49 years; 50-59 years; 60-69 years; and 70-91 years. Inter-correlations between age, \%IPF and A-IPF were computed through the Spearman's correlation which was used to assess the pairwise relationship between the variables. $P$ values $\leq 0.05$ were considered statistically significant. All data is expressed as 2.5th and 97.5th percentiles (central 95\% percentiles) unless stated otherwise.

\section{Results}

A total of 2366 blood samples were collected from individuals with ages range from 16 to 91 years (765 males and 1527 females). 74 outliers were removed and the remaining 2292 samples were included in the data analysis. The distribution of both \%-IPF and A-IPF (including the subgroups, data not shown) was non-Gaussian (non-parametric data with $P<0.05$ ), showing left skewed histograms (Figure 2A and 2B) using visual examination. Unfortunately, it was not possible to repeat outlying \%-IPF and A-IPF results due to the temporal requirements of the study design.

This non-Gaussian distribution was confirmed by the Kolmogorov-Smirnov Z-test [40], which was used to test all variables for normality. A non-parametric distribution was shown in all subgroups, thus the Kruskal-Wallis rank sum test, Mann-Whitney U test, and Spearman's rank-order correlation were used to analyse the data.

The reference interval data for \%-IPF and A-IPF for total individuals and sex-divided subgroups are presented in Table 1. The reference interval for \%-IPF was 1.6-10.1\% (median \%-IPF, 4.4\%; median age, 41 years) in total individuals: $1.8-10.0 \%$ in males and $1.5-10.1 \%$ in females. The reference interval for A-IPF was 4.37-23.21 x 10%/ (median A-IPF, 10.88 x 10\%/l; median age, 41 years) in total individuals: 4.66-22.19 x $10^{9} / 1$ in males and $4.36-23.49 \times 10^{9} / 1$ in females. 
There was no statistical difference in the reference intervals between males and females for A-IPF $(P=0.373)$. Likewise, the Kruskal-Wallis rank sum test showed that there was no overall statistical significance amongst the seven age subgroups for both \%-IPF $(P=0.1186)$ and A-IPF $(P=0.3823)$, respectively. Furthermore, a statistical exploration of the correlation between age versus \%-IPF Spearman $\rho=0.0156(P=0.454)$, and age versus A-IPF - Spearman $\rho=-0.0023(P=0.9115)$, showed a statistically insignificant and substantially uncorrelated relationship (Figure 4). However, as shown in Figures 3 and 4, respectively, the two positive results this study yielded are a statistical difference in the reference intervals between males and females for \%-IPF $(P=0.004)$ and the relationship between \%-IPF and A-IPF which was statistically significant and highly correlated, Spearman $\rho=0.909(P<0.001)$.

\section{Discussion}

After decades of technical challenges in enumerating immature platelets, a real-time IPF diagnostic tool is available on several automated Sysmex haematology analytical platforms. The clinical utility of this tool is reported in several conditions [4-27]. Of these, the role of immature platelets to indicate the mechanism of thrombocytopenia is well recognised [4-10] due to its ability to differentiate central and peripheral thrombocytopenias [31]. In 2012, an expert group published recommendations for the management of thrombocytopenia and strongly agreed that decisions to treat thrombocytopenia should consider, amongst other factors, mechanisms of thrombocytopenia [43]. To this end, IPF has the potential to offer insight into the underlying mechanisms of thrombocytopenia, although there is no fundamental UK-based reference interval data currently available for IPF on the Sysmex XN-series analysers for the purpose of future research and potential clinical use. 
Historically, IPF reference intervals were determined using Sysmex predecessors (Sysmex XE2100 or XE-5000) or by using the XN-series analysers comprising individuals from a different population group [29,34]. Since the Sysmex XN-series utilises different principles for IPF measurement from its previous version [44], it is important to investigate whether the reference intervals established on the previous version are valid using the $\mathrm{XN}$-series analysers. A slight difference was observed between reference intervals across a number of studies (Table 2) using a range of Sysmex haematology platforms. The reference intervals obtained on the Sysmex XNseries were generally higher and demonstrated a wider distribution for both \%-IPF and A-IPF compared to the measurement attained on the previous instruments (XE-series analysers) $[29,31,34,45-54]$.

In this study, we measured IPF in a large number of samples (total $n=2366$, aged $\geq 16$ years old) with FBC parameters reported within population specific reference ranges. This is the largest study conducted to date for determining the reference intervals for \%-IPF and A-IPF (Table 2). The reference interval for \%-IPF and A-IPF was 1.6-10.1\% and 4.37-23.21 x 10\% / respectively, when specimens from both sexes were combined. However, a statistically significant difference between sexes was apparent and therefore this study established separate reference intervals for sex-specific subgroups for \%-IPF - viz. 1.8-10.0\% for males and 1.5-10.1\% for females.

Correlation analysis was also performed in this study; a biological relationship between age and \%-IPF or A-IPF is highly unlikely in view of i) the extremely low coefficients of correlation (Spearman $\rho=0.0156$ and $\rho=0.0023)$, ii) a rather low statistical significance $(P=0.454$ and 0.9115), and iii) the large number of data analysed $(n=2292)$. In contrast, a positive association 
between \%-IPF and A-IPF was confirmed in a highly significant manner, as Figure 4 demonstrates, with a Spearman $\rho=0.909(P<0.001)$. This was expected as $\%$-IPF values are used to calculate A-IPF, and therefore, a mathematical inter-relatedness exists between the two parameters.

Overall, the reference intervals achieved in this study compared well with previous studies, especially for the same series of Sysmex instruments[29,34]. There was a larger variation reported for \%-IPF and A-IPF between our study and those conducted on earlier instruments, most of which were considerably narrower than our reference intervals. This variation between studies may represent differences in: the platforms used, populations studied, and methodologies adopted for determining reference intervals and excluding outliers. Therefore, a standardised application of this parameter in a clinical setting has been somewhat difficult [55] and a need for instrument-specific reference intervals is vindicated.

The present study has three limitations. Firstly, the XN-10 used an automated switching algorithm to report the most accurate platelet count (PLT-I, PLT-O, or PLT-F). This was then used to calculate the A-IPF instead of setting fluorescent platelets (PLT-F) as a fixed method for this purpose. This may have slightly increased the A-IPF values because the PLT-I and PLT-O are known to generate higher platelet counts compared to the PLT-F [56]. Secondly, this study did not stratify reference intervals according to ethnicity and clinical situations. This may restrict the application of these reference intervals in different ethnic populations and may impact on its value in the clinical setting. Thirdly, the influence of biological variation that is essential for analytical specifications and estimating reference change values was not verified in this study. This may limit the clinical interpretation of \%-IPF and A-IPF [57] and therefore further studies are warranted to investigate the effects of biological variations. 


\section{Conclusion}

We have generated reference intervals for both A-IPF and \%-IPF in a UK population and found that for \%-IPF there are sex-specific reference intervals, $1.8-10.0 \%$ for males and $1.5-10.1 \%$ for females. Reference intervals for A-IPF are independent of sex and likewise reference intervals for both \%-IPF and A-IPF are independent of age. There was a strong correlation between \%-IPF and A-IPF which was expected due to the inter-relatedness of these parameters.

Word Count: 2624

\section{Tables with their headings:}

Table 1 Reference intervals for \%-IPF and A-IPF on Sysmex XN-10 in samples from apparently healthy individuals.

\begin{tabular}{|c|c|c|c|c|c|c|c|c|c|c|}
\hline & \multicolumn{3}{|c|}{$\mathbf{n}$} & Total & \multicolumn{3}{|c|}{ Males } & \multicolumn{3}{|c|}{ Females } \\
\hline & T & M & F & RI & RI & Median & $\begin{array}{c}\text { Age } \\
\text { Range }\end{array}$ & RI & Median & $\begin{array}{c}\text { Age } \\
\text { Range }\end{array}$ \\
\hline \%-IPF, \% & 2292 & 765 & $1527^{\mathrm{a}}$ & $1.6-10.1^{c}$ & $1.8-10.0$ & 4.6 & $16-91^{\mathrm{e}}$ & $1.5-10.1$ & 4.3 & $16-90^{\dagger}$ \\
\hline A-IPF, x 109/I & 2292 & 765 & $1527^{b}$ & $4.37-23.21^{\mathrm{d}}$ & $4.66-22.19$ & 10.71 & $16-91^{\mathrm{e}}$ & $4.36-23.49$ & 10.97 & $16-90^{f}$ \\
\hline
\end{tabular}

A-IPF, absolute immature platelet fraction; \%-IPF, percentage immature platelet fraction; T, total; M, males; F, females; n, sample size; and RI, reference interval. ${ }^{a} P=0.004$ vs. males (MannWhitney U-test), decision $=$ rejectnull hypothesis; ${ }^{\mathrm{b}} \mathrm{P}=0.373$ vs. males (Mann-Whitney U-test), decision = accept null hypothesis; ${ }^{\mathrm{c}}$ Median, 4.4\%; ${ }^{\mathrm{d}}$ Median, $10.88 \times 10^{9} / \mathrm{l}$; ${ }^{\mathrm{e}}$ Median, 42 years; and ${ }^{\mathrm{f}}$ Median, 40 years. The data are expressed as 2.5th and 97.5th percentiles except for age range which is expressed as a whole range. 
Table 2 Comparison of reference intervals for A-IPF and \%-IPF using Sysmex analytical platforms from 2004 to date.

\begin{tabular}{|c|c|c|c|c|c|c|c|}
\hline Instrument & Population & $\mathbf{N}$ & $\operatorname{sex}$ & \%-IPF & $\begin{array}{l}\text { A-IPF } \\
\left(\times 10^{9} / 1\right)\end{array}$ & Author & $\begin{array}{c}\text { Year of } \\
\text { publication }\end{array}$ \\
\hline Sysmex XE-2100 & UK & 50 & Male/Female & $1.1-6.1$ & - & Briggs $^{[31]}$ & 2004 \\
\hline Sysmex XE-5000 & German & 309 & Male/Female & $0.8-6.3$ & $2.3-12.7$ & Pekelharing ${ }^{[53]}$ & 2010 \\
\hline Sysmex XE-2100 & South African & 60 & Male/Female & $0.7-5.5$ & - & Mogongoa $^{[54]}$ & 2012 \\
\hline Sysmex XE-5000 & Brazil & 132 & Male/Female & $0.8-5.6$ & - & Morkis ${ }^{[45]}$ & 2016 \\
\hline Sysmex XE-2100 & Japan & 82 & Male/Female & $0.5-5.7$ & $1.4-10.4$ & Takami ${ }^{[48]}$ & 2007 \\
\hline Sysmex XE-2100 & South Korea & 2152 & Male/Female & $0.5-3.3$ & $1.25-7.02$ & $\mathrm{Ko}^{[49]}$ & 2013 \\
\hline Sysmex XE-2100 & USA & 28 & Not stated & $0.8-10.1$ & $2.1-19.6$ & Berny-Lang ${ }^{[46]}$ & 2015 \\
\hline Sysmex XE-2100 & India & 945 & Male/Female & $0.3-8.7$ & - & Sachdev ${ }^{[50]}$ & 2014 \\
\hline Sysmex XE-2100 & Italy & 229 & Male/Female & $1.9-4.1$ & $4.2-8.2$ & Cesari $^{[51]}$ & 2013 \\
\hline Sysmex XE-2100 & India & 100 & Not stated & $0.7-4.3$ & - & $\operatorname{Dadu}^{[52]}$ & 2014 \\
\hline Sysmex XE-5000 & Denmark & 1674 & Male/Female & $1.3-9.0$ & - & Joergensen ${ }^{[47]}$ & 2016 \\
\hline Sysmex XN9000 & China & 2179 & Male/Female & $0.4-10.8$ & $0.8-23.0$ & Yang ${ }^{[29]}$ & 2017 \\
\hline $\begin{array}{l}\text { Sysmex XN and } \\
\text { Sysmex XE-2100 }\end{array}$ & Korea & 2104 & Male/Female & $\begin{array}{l}\text { XN: } 1.0-7.3 \\
\text { XE: } 0.5-3.3\end{array}$ & $\begin{array}{l}\text { XN: } 2.49-15.64 \\
\text { XE: } 1.25-7.02\end{array}$ & $\mathrm{Ko}^{[34]}$ & 2015 \\
\hline Sysmex XN9000 & UK & 2366 & Male/Female & $\begin{array}{c}\text { Total: } 1.6-10.1 \\
\text { Males: } 1.8-10.0 \\
\text { Females: } 1.5-10.1\end{array}$ & Total: 4.37-23.21 & Current study & 2017 \\
\hline
\end{tabular}


Figures:

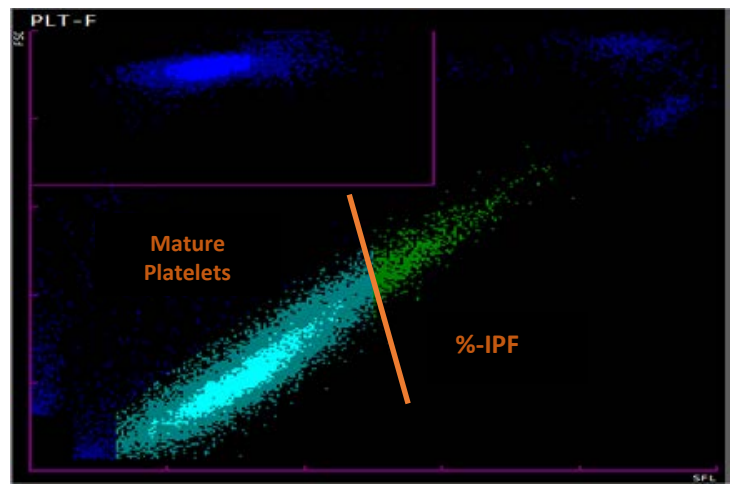

Figure 1

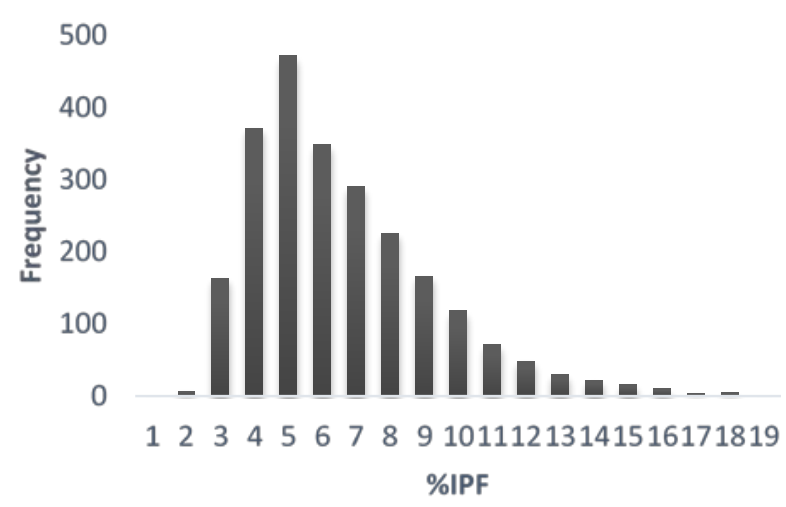

Figure 2A

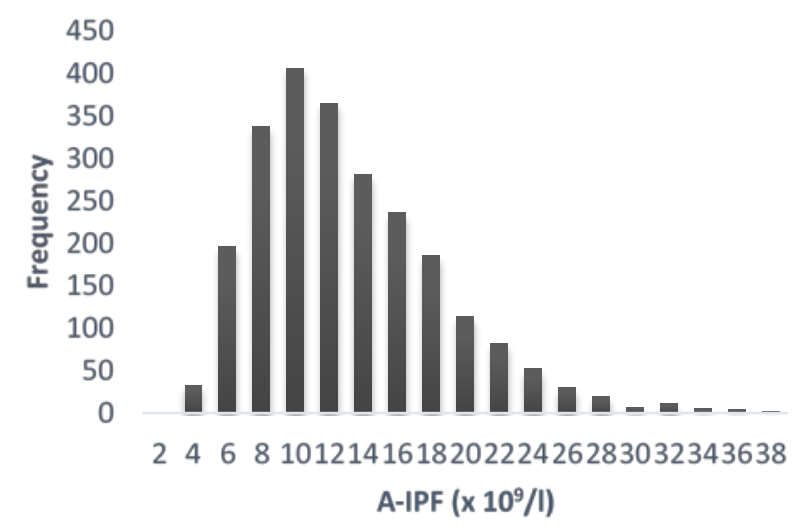

Figure 2B 


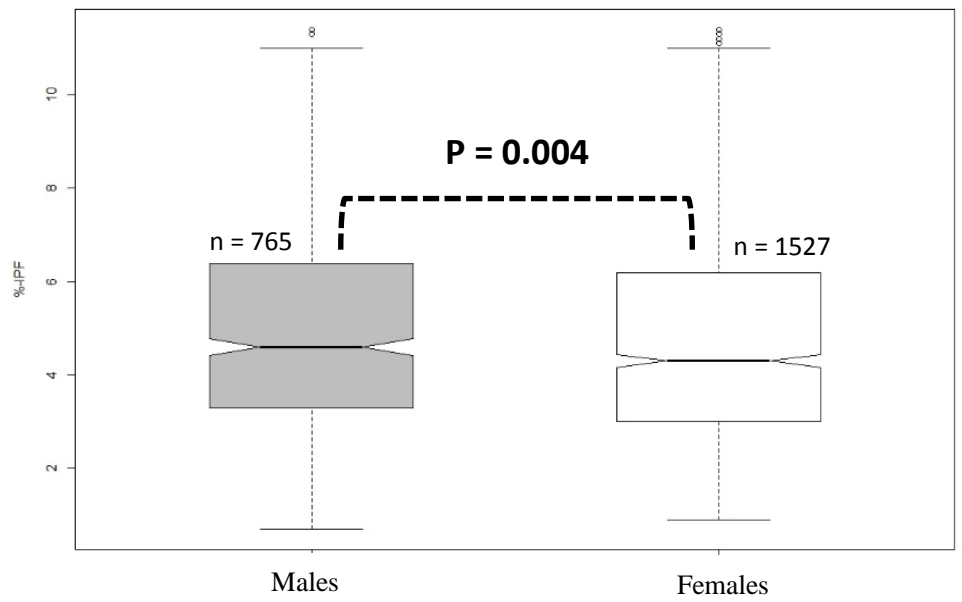

Figure 3

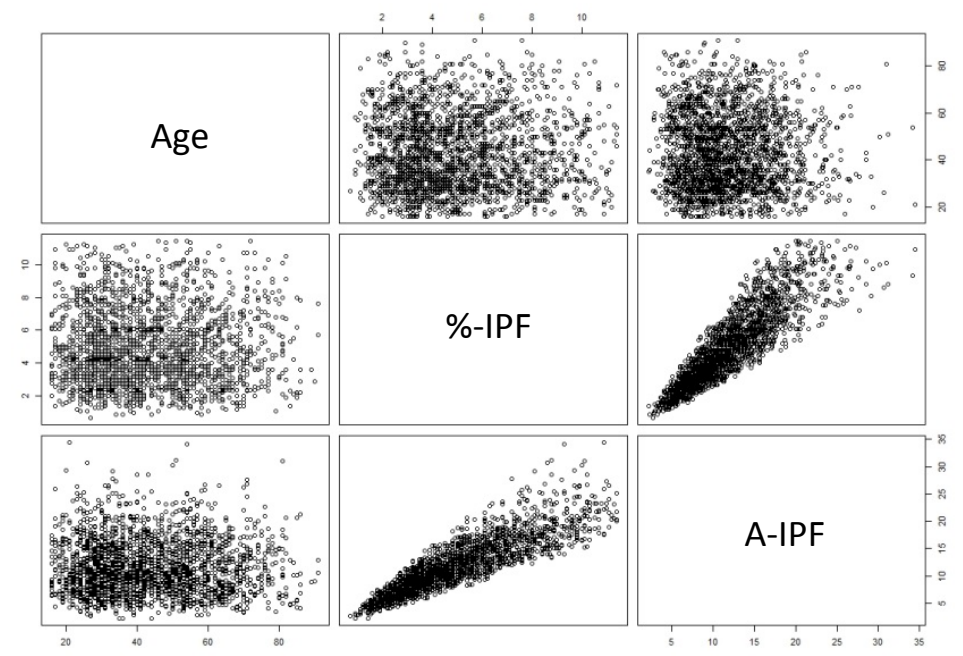

Figure 4 


\section{Figure Legends:}

Figure 1. Example of IPF scattergram of PLT-F channel on Sysmex XN-10 showing fluorescence intensity (RNA content) and forward scatter (size of the platelets)

Figure 2. A. Frequency distribution of \%-IPF $(n=2366)$. B. Frequency distribution of A-IPF $(n=2366)$.

Figure 3. Boxplot of \%-IPF data for sex subgroups $(\mathrm{n}=2292)$.

Figure 4. Scatter plot matrix illustrating all possible pairwise relationships between variables (age, \%-IPF and A-IPF). Age, years; \%-IPF, \%; A-IPF, x 10\% /. Spearman's rank correlation rho between age and \%-IPF, $\rho=0.0157(P=0.454)$; Spearman's rank correlation rho between age and A-IPF, $\rho=$ -0.0023 $(P=0.9115)$; and Spearman's rank correlation rho between \%-IPF and A-IPF, $\rho=0.909(P$ $<0.001)$ 


\section{References}

[1] Ingram, M, Coopersmith, A. Reticulated platelets following acute blood loss. Br J Haematol $1969 ; 17: 225-29$.

[2] Lee LG, Chen CH, Chiu LA. Thiazole orange: a new dye for reticulocyte analysis. Cytometry $1986 ; 7: 508-17$.

[3] Kienast J, Schmitz G. Flow cytometric analysis of thiazole orange uptake by platelets: a diagnostic aid in the evaluation of thrombocytopenic disorders. Blood 1990;75:116-21.

[4] Rinder HM, Munz UJ, Ault KA, Bonan JL, Smith BR. Reticulated platelets in the evaluation of thrombopoietic disorders. Arch Pathol Lab Med 1993;117:606-10.

[5] Ault KA, Rinder HM, Mitchell J, Carmody MB, Vary CP, Hillman RS. The significance of platelets with increased RNA content (reticulated platelets): a measure of the rate of thrombopoiesis. Am J Clin Pathol 1992;98:637-46.

[6] Richards EM, Baglin TP. Quantitation of reticulated platelets: methodology and clinical application. Br J Haematol 1995;91:445-51.

[7] Watanabe K, Takeuchi K, Kawai Y, Ikeda Y, Kubota F, Nakamoto H. Automated measurement of reticulated platelets in estimating thrombopoiesis. Eur J Haematol 1995;54:163-71.

[8] Koike Y, Yoneyama A, Shirai J, Ishida T, Shoda E, Miyazaki K, Sunaga S, Horie R, Aoki K, Koike K, Ogata I, Tahara T, Kato T, Nakahara K, Kariya T, Higashihara M. Evaluation of thrombopoiesis in thrombocytopenic disorders by simultaneous measurement of reticulated platelets of whole blood and serum thrombopoietin concentrations. Thromb Haemost 1998;79:1106-10.

[9] Saxon BR, Blanchette VS, Butchart S, Lim-Yin J, Poon AO. Reticulated platelet counts in the diagnosis of acute immune thrombocytopenic purpura. J Pediatr Hematol Oncol 1998;20:44-8. 
[10] Saxon BR, Mody M, Blanchette VS, Freedman J. Reticulated platelet counts in the assessment of thrombocytopenic disorders. Acta Paediatr 1998;424(Suppl):65-70.

[11] Rinder HM, Schuster JE, Rinder CS, Wang C, Schweidler HJ, Smith BR. Correlation of thrombosis with increased platelet turnover in thrombocytosis. Blood 1998;91:1288-94.

[12] Robinson MS, Harrison C, Mackie IJ, Machin SJ, Harrison P. Reticulated platelets in primary and reactive thrombocytosis. Br J Haematol 1998;101:388-9.

[13] Joseph JE, Donohoe S, Harrison P, Mackie IJ, Machin SJ. Platelet activation and turnover in the primary antiphospholipid syndrome. Lupus 1998;7:333-40.

[14] Harrison P. Platelet function and turnover in acute coronary syndromes. Blood 2000;96:256A. [15] Semple JW, Siminovitch KA, Mody M, Milev Y, Lazarus AH, Wright JF, Freedman J. Flow cytometric analysis of platelets from children with the Wiskott-Aldrich syndrome reveals defects in platelet development, activation and structure. Br J Haematol 1997;97:747-54.

[16] Fabris F, Cordiano I, Steffan A, Ramon R, Scandellari R, Nichol JL, Girolami A. Indirect study of thrombopoiesis (TPO, reticulated platelets, glycocalicin) in patients with hereditary macrothrombocytopenia. Eur J Haematol 2000;64:151-6.

[17] Romp KG, Peters WP, Hoffman M. Reticulated platelet counts in patients undergoing autologous bone marrow transplantation: an aid in assessing marrow recovery. Am J Hematol 1994;46:319-24.

[18] Richards EM, Jestice HK, Mahendra P, Scott MA, Marcus RE, Baglin TP. Measurement of reticulated platelets following peripheral blood progenitor cell and bone marrow transplantation: implications for marrow reconstitution and the use of thrombopoietin. Bone Marrow Transplant 1996;17:1029-33. 
[19] Catani L, Vianelli N, Luatti S, Rizzi S, Castellani S, Valdrè L, Lemoli RM, Tura S. Characterization of autotransplant-related thrombocytopenia by evaluation of glycocalicin and reticulated platelets. Bone Marrow Transplant 1999;24:1191-4.

[20] Stohlawetz P, Stiegler G, Knöbl P, Höcker P, Panzer S. The rise of reticulated platelets after intensive chemotherapy for AML reduces the need for platelet transfusions. Ann Hematol 1999;78:271-3.

[21] Stiegler G, Stohlawetz P, Brugger S, Jilma B, Vierhapper H, Höcker P, Panzer S. Elevated numbers of reticulated platelets in hyperthyroidism: direct evidence for an increase of thrombopoiesis. Br J Haematol 1998;101:656-8.

[22] Tassies D, Reverter JC, Cases A, Escolar G, Villamor N, Lopez-Pedret J, Castillo R, Ordinas A. Reticulated platelets in uremic patients: effect of hemodialysis and continuous ambulatory peritoneal dialysis. Am J Hematol 1995;50:161-6.

[23] Himmelfarb J, Holbrook D, McMonagle E, Ault K. Increased reticulated platelets in dialysis patients. Kidney Int 1997;51:834-9.

[24] Tassies D, Reverter JC, Cases A, Calls J, Escolar G, Ordinas A. Effect of recombinant human erythropoietin treatment on circulating reticulated platelets in uremic patients: association with early improvement in platelet function. Am J Hematol 1998;59:105-9.

[25] Rinder HM, Bonan JL, Anandan S, Rinder CS, Rodrigues PA, Smith BR. Noninvasive measurement of platelet kinetics in normal and hypertensive pregnancies. Am J Obstet Gynecol 1994;170:117-22.

[26] Joseph MA, Adams D, Maragos J, Saving KL. Flow cytometry of neonatal platelet RNA. J Pediatr Hematol Oncol 1996;18:277-81.

[27] Peterec SM, Brennan SA, Rinder HM, Wnek JL, Beardsley DS. Reticulated platelet values in normal and thrombocytopenic neonates. J Pediatr 1996;129:269-74. 
[28] Hoffmann JJ. Reticulated platelets: analytical aspects and clinical utility. Clin Chem Lab Med 2014;52:1107-17.

[29] Yang B, Zhou Y, Ding S, Zha H, Zhang F, He W, Xu Y. Establishment of reference interval for immature platelet fraction using Sysmex XN9000 hematology analyzer in Anhui province in China. Int J Clin Exp Med 2017;10:4214-20.

[30] Briggs C, Harrison P, Grant D, Staves J, MacHin SJ. New quantities parameters on a recently introduced automated blood cell counter— the XE-2100. Clin Lab Haem 2000;22:345-50.

[31] Briggs C, Kunka S, Hart D, Oguni S, Machin SJ. Assessment of an immature platelet fraction (IPF) in peripheral thrombocytopenia. Br J Haematol 2004;126:93-9.

[32] Briggs C, Longair I, Kumar P, Singh D, Machin SJ. Performance evaluation of the Sysmex haematology XN modular system. J Clin Pathol 2012;65:1024-30.

[33] Cremer M. The immature platelet fraction (IPF) in neonates. Diagnostic Perspectives 2011;1:36-42.

[34] Ko YJ, Hur M, Kim H, Choi SG, Moon HW, Yun YM. Reference interval for immature platelet fraction on Sysmex XN hematology analyzer: a comparison study with Sysmex XE-2100. Clin Chem Lab Med 2015;53:1091-7.

[35] Meintker L, Haimerl M, Ringwald J, Krause SW. Measurement of immature platelets with Abbott CD-Sapphire and Sysmex XE-5000 in haematology and oncology patients. Clin Chem Lab Med 2013;51:2125-31.

[36] Karpatkin S. Human platelet senescence. Annu Rev Med 1972;23:101-28.

[37] Harrison P, Goodall AH. Message in the platelet - more than just vestigial mRNA! Platelets 2008;19:395-404. 
[38] Barsam SJ, Psaila B, Forestier M, Page LK, Sloane PA, Geyer JT, Villarica GO, Ruisi MM, Gernsheimer TB, Beer JH, Bussel JB. Platelet production and platelet destruction: assessing mechanisms of treatment effect in immune thrombocytopenia. Blood 2011;117:5723-32.

[39] Solberg HE, Lahti A. Detection of outliers in reference distributions: performance of Horn's algorithm. Clin Chem 2005;51:2326-32.

[40] Reed AH, Henry RJ, Mason WB. Influence of statistical method used on the resulting estimate of normal range. Clin Chem 1971;17:275-84.

[41] Clinical and Laboratory Standards Institute. Defining, Establishing, and Verifying Reference Intervals in the Clinical Laboratory; Approved Guideline - 3rd Edition (EP28-A3c) Horowitz, GL: CLSI, 2010.

[42] Roehrl MH, Lantz D, Sylvester C, Wang JY. Age-Dependent Reference Ranges for Automated Assessment of Immature Granulocytes and Clinical Significance in an Outpatient Setting. Arch Pathol Lab Med 2011;135(4):471-7.

[43] Van der Linden T, Souweine B, Dupic L, Soufir L, Meyer P. Management of thrombocytopenia in the ICU (pregnancy excluded). Ann Intensive Care 2012;2:42.

[44] Kim H, Hur M, Choi SG, Moon HW, Yun YM, Hwang HS, Kwon HS, Sohn IS. Performance evaluation of Sysmex XN hematology analyzer in umbilical cord blood: a comparison study with Sysmex XE-2100. Clin Chem Lab Med 2014;52:1771-9.

[45] Morkis IV, Farias MG, Scotti L. Determination of reference ranges for immature platelet and reticulocyte fractions and reticulocyte hemoglobin equivalent. Rev Bras Hematol Hemoter 2016;38:310-3. 
[46] Berny-Lang MA, Darling CE, Frelinger AL, Barnard MR, Smith CS, Michelson AD. Do immature platelet levels in chest pain patients presenting to the emergency department aid in the diagnosis of acute coronary syndrome? Int J Lab Hematol 2015;37:112-9.

[47] Joergensen MK, Bathum L. Reference intervals for mean platelet volume and immature platelet fraction determined on a sysmex XE5000 hematology analyzer. Scand J Clin Lab Invest 2016;76:172-6.

[48] Takami A, Shibayama M, Orito M, Omote M, Okumura H, Yamashita T, Shimadoi S, Yoshida T, Nakao S, Asakura H. Immature platelet fraction for prediction of platelet engraftment after allogeneic stem cell transplantation. Bone Marrow Transplantation 2007;39:501-7.

[49] Ko YJ, Kim H, Hur M, Choi SG, Moon HW, Yun YM, Hong SN. Establishment of reference interval for immature platelet fraction. Int J Lab Hematol 2013;35:528-33.

[50] Sachdev R, Tiwari AK, Goel S, Raina V, Sethi M. Establishing biological reference intervals for novel platelet parameters (immature platelet fraction, high immature platelet fraction, platelet distribution width, platelet large cell ratio, platelet-X, plateletcrit, and platelet distribution width) and their correlations among each other. Indian J Pathol Microbiol 2014;57:231-5.

[51] Cesari F, Marcucci R, Gori AM, Caporale R, Fanelli A, Casola G, Balzi D, Barchielli A, Valente S, Giglioli C, Gensini GF, Abbate R. Reticulated platelets predict cardiovascular death in acute coronary syndrome patients. Insights from the AMI-Florence 2 Study. Thromb Haemost 2013;109:846-53.

[52] Dadu T, Sehgal K, Joshi M, Khodaiji S. Evaluation of the immature platelet fraction as an indicator of platelet recovery in dengue patients. Int J Lab Hematol 2014;36:499-504.

[53] Pekelharing JM, Hauss O, De Jonge R, Lokhoff J, Sodikromo J, Spaans M, Brouwer R, De Lathouder S, Hinzmann R. Haematology reference intervals for established and novel parameters in healthy adults. Sysmex Diagnostic Perspectives 2010;1:1-11. 
[54] Mogongoa LF, Botma J, Jaftha AD, van Rensburg WJ. Reference ranges for platelet indices using Sysmex XE-2100 blood analyser. Medical Technology SA 2012;26:17-21.

[55] Yamaoka G, Kubota Y, Nomura T, Inage T, Arai T, Kitanaka A, Saigo K, Iseki K, Baba N, Taminato $\mathrm{T}$. The immature platelet fraction is a useful marker for predicting the timing of platelet recovery in patients with cancer after chemotherapy and hematopoietic stem cell transplantation. Int J Lab Hematol 2010;32:e208-16.

[56] Schoorl M, Schoorl M, Oomes J, van Pelt J. New fluorescent method (PLT-F) on Sysmex XN2000 hematology analyzer achieved higher accuracy in low platelet counting. Am J Clin Pathol 2013;140:495-9.

[57] Buoro S, Seghezzi M, Manenti B, Pacioni A, Carobene A, Ceriotti F, Ottomano C, Lippi G. Biological variation of platelet parameters determined by the Sysmex XN hematology analyzer. Clinica Chimica Acta 2017;470:125-32.

\section{Acknowledgements}

The authors would like to thank the staff at the Department of Haematology at Homerton University Hospital NHS Foundation Trust for their assistance during this project. Author contributions: UA designed the project and wrote the paper, RG, GK and DAT co-designed the project and critically reviewed the manuscript.

\section{Disclosure of conflict of interest: The authors have no conflict of interest to declare.}

